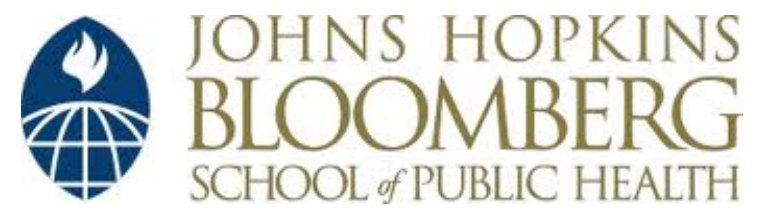

Johns Hopkins University, Dept. of Biostatistics Working Papers

9-20-2004

\title{
A Hypothesis Test for the End of a Common Source Outbreak
}

\author{
Ron Brookmeyer \\ Johns Hopkins Bloomberg School of Public Health, Department of Biostatistics, rbrookmeyer@ucla.edu \\ Xiaojun You \\ Johns Hopkins Bloomberg School of Public Health, Department of Biostatistics
}

\section{Suggested Citation}

Brookmeyer, Ron and You, Xiaojun, "A Hypothesis Test for the End of a Common Source Outbreak" (September 2004). Johns Hopkins University, Dept. of Biostatistics Working Papers. Working Paper 60.

http://biostats.bepress.com/jhubiostat/paper60

This working paper is hosted by The Berkeley Electronic Press (bepress) and may not be commercially reproduced without the permission of the copyright holder.

Copyright $(\mathcal{C} 2011$ by the authors 


\title{
A Hypothesis Test for the End of a Common Source Outbreak
}

\author{
Ron Brookmeyer \\ Department of Biostatistics \\ Johns Hopkins Bloomberg School of Public Health \\ Baltimore, Maryland 21205, U.S. \\ Email: rbrook@jhsph.edu \\ and \\ Xiaojun You \\ Department of Biostatistics \\ Johns Hopkins Bloomberg School of Public Health
}




\section{SUMMARY}

The objective of this paper is to develop a hypothesis testing procedure to determine whether a common source outbreak has ended. We do not assume that the calendar date of exposure to the pathogen is known. We assume an underlying parametric model for the incubation period distribution of a 2-paramter exponential model with a guarantee time, although the parameters are not assumed to be known. The hypothesis testing procedure is based on the spacings between ordered calendar dates of disease onset of the cases. A simulation study was performed to evaluate the robustness of the methods to a lognormal model for the incubation period of infectious diseases. We investigated whether multiple testing over the course of the outbreak could increase the overall outbreak-wise type 1 error probability. We derive expressions for the outbreak-wise type 1 error probability and show that multiple testing has minimal effect on inflating that error probability.

KEY WORDS: Epidemic; Hypothesis test; Infectious disease; Spacing 


\section{Introduction}

During the course of a disease outbreak, public health officials may be asked by media and the public whether or not the outbreak is over. For example, during the 2001 anthrax outbreak in the United States, the media sought reassurance for the public that the outbreak had ended and asked public health officials if they expected any additional cases. In response, public health officials observed that there had been no new cases in several days, and suggested that the lull in cases provided some evidence that the outbreak was waning if not completely over. One official suggested that if no new cases occurred after $t$ days had elapsed, then the epidemic was likely over. However, no theoretical justification was given for that statement or for the specific value for $t$. An objective of this paper is to investigate if there is any justification for such a methodology and under what conditions, and when such an approach can be misleading. Public health officials need simple real time methodological tools to help address the question as to whether or not a common source outbreak is over. In this paper we define a common source outbreak as one in which there is a single exposure that occurs at a single point in calendar time for all cases. For example, the 2001 U.S. outbreak among workers in a postal facility was a common source outbreak because the exposure occurred during a brief period of time when a letter contaminated with anthrax spores was processed in the facility (Brookmeyer and Blades, 2002). The assumption of a common source outbreak may also apply to bioterrorism threats other than anthrax such as tularemia in which the agent could be aerosolized and exposure occurs in a brief period of time. We are not considering in this paper outbreaks that are propagated by person to person transmission.

If both the incubation period distribution of disease and the time of exposure to the infectious source (the pathogen) are known, the ultimate size of an outbreak can be estimated in a very 
straightforward way. The incubation period of disease is the time from exposure to the pathogen to onset of disease. The basic idea is that the earliest cases that occur are the ones with the shortest incubation period. Thus, the observed number of cases represents only a fraction of the total cases that will eventually occur and the fraction is determined by the incubation period distribution. For example, if the median incubation period is 10 days and 7 cases have occurred by day 10 following exposure then that suggests the total outbreak size $(N)$ is about $7 / 0.5=14$ cases. This simple calculation method can be generalized to handle the more complex situation when the calendar date of exposure is unknown. These more general methods have been termed back-calculation methods (Brookmeyer and Gail, 1994). Back-calculation methods can account for situations when exposure to the pathogen occurs at multiple discrete time points or continuously in time. Recently, Walden and Kaplan (2004) cast the problem in a Bayesian framework to the real-time estimation of the size and exposure date of an outbreak by incorporating a prior on the size of the outbreak $(N)$. However, all of these methods require knowledge of the incubation period distribution.

The goal of this paper is to consider the situation when neither the incubation period distribution nor the time of exposure is known. We sought to provide a statistical framework for addressing the question public health officials faced in the 2001 anthrax outbreak: can a lull of cases provide evidence that an epidemic is over, or could the lull be misleading. In section 2, we formulate the problem in terms of a hypothesis test. In statistical terms, the lull between cases is described by the spacings between order statistics. There has been considerable theoretical development on the spacings of order statistics (Pyke, 1965; David, 1970; Cox and Hinkley, 1974). We use the theory of spacings of order statistics to address the question of whether a common source outbreak is over. The impact of multiple testing on the overall outbreak-wise 
probability of a type 1 error is discussed in section 3. A simulation study to investigate the properties of the procedures is reported in section 4 . The results are discussed in section 5 .

\section{A Hypothesis Test to Determine if a Common Source Outbreak is Over}

\subsection{Formulation}

Let $y_{i}$ be the ordered calendar dates of disease onset of the $i^{\text {th }}$ case; that is $y_{l}$ is the earliest onset date, $y_{2}$ is the second earliest and so on. Suppose we have observed $n$ cases and their calendar dates of onset are $y_{1}, \ldots y_{n}$. Further, suppose that $t$ days have passed since the last case $\left(y_{n}\right)$ occurred. We wish to address the question as to whether there is any evidence that the outbreak is over from this limited data.

The objective is to develop a hypothesis testing procedure if neither the exposure date to the pathogen nor the incubation period distribution is known. Suppose the ultimate size of the common source outbreak is $N$ which is considered an unknown parameter; that is, $N$ cases of disease would eventually occur. At a point in time, suppose we have observed $n$ of the $N$ cases. The question is, are there more cases still to come? We want to control the type 1 error of falsely declaring the epidemic is over and communicating a false sense of security. Thus, we consider the null hypothesis that the epidemic is not over, that is, $N>n$ versus the alternative hypothesis that the epidemic is over, that is, $N=n$. Thus,

$$
\begin{aligned}
& \mathrm{H}_{0}: N>n \\
& \mathrm{H}_{1}: N=n
\end{aligned}
$$

One tempting approach is to obtain the maximum likelihood estimator of $N$ from a full likelihood. However, the difficulties with this approach become immediately apparent even with the simple exponential model for the incubation period. Suppose the probability density of the incubation period, $f(u)$, is exponential $f(u)=\lambda \exp (-\lambda u)$ with hazard rate $\lambda$ and mean $\mu=\lambda^{-1}$. 
Then, it can be shown that if both the calendar date of exposure to the pathogen $(E)$ and the size of the outbreak $(N)$ are unknown parameters, then the maximum likelihood estimator of $N$ is $\hat{N}=n$. Thus, the maximum likelihood estimator of $N$ is always the number of cases observed to date. Similar difficulties with the likelihood based approach for the lognormal distribution have been reported by Hill (1963), Giesbrecht and Kempthorne (1976), and other investigators. In the next section, we develop an approach based on the spacings or lags between the occurrences of cases.

\subsection{Hypothesis Test Based on Spacings}

We use an approach based on the spacings between calendar dates of disease onset. We define the spacings as the difference between the successive ordered calendar dates of onset. Specifically, the $j^{\text {th }}$ spacing is $s_{j}=y_{j}-y_{j-1}$. Thus, from the first $n$ calendar dates of disease onset we can obtain the first $n-1$ spacings.

The calendar date of disease onset $y$ is related to the incubation period $u$ and the calendar date of exposure $(E)$ through $y=E+u$. Thus, the $j^{\text {th }}$ spacing of the calendar dates of disease onset is $s_{j}=y_{j}-y_{j-1}=u_{j}+E-u_{j-1}-E=u_{j}-u_{j-1}$ which is also the $j^{\text {th }}$ spacing of the incubation periods. Accordingly, the probability distribution of the spacings depends on the incubation period distribution and $N$ but does not depend on the calendar date of exposure $E$.

We begin by assuming that the incubation period distribution follows a two parameter exponential model with a guarantee time, that is, the incubation period density is $f(u)=\lambda \exp (-\lambda u)$ for $u>G$, and 0 for $u<G$. The guarantee time parameter requires that all incubation periods are greater than $G$ days, and introduction of the guarantee times leads to a much more flexible family to describe the incubation period of infectious diseases than the one parameter exponential model. We will not assume that the parameters $\lambda$ or $G$ are known. The 
exponential model has been found to be useful to describe the incubation period of anthrax based both on mechanistic and empirical considerations (Brookmeyer, Johnson and Bollinger, 2003; Brookmeyer, Johnson and Barry, 2004).

The $j^{\text {th }}$ spacing arising from a sample of size $N$ from a one parameter exponential model with hazard rate $\lambda$ is known to have an exponential distribution with hazard rate $\lambda(N-j)$ and furthermore, the spacings are independent (David, 1970; Cox and Hinkely, 1974). It follows immediately that the $j^{\text {th }}$ spacing arising from a sample of size $N$ from the two parameter exponential model (with guarantee time $G$ and hazard rate $\lambda$ ) also has an exponential distribution with parameter $\lambda(N-j)$, that is, the pdf of the $j^{\text {th }}$ spacing is

$$
f\left(s_{j}\right)=\lambda(N-j) \exp \left(-\lambda(N-j) s_{j}\right)
$$

Suppose $n$ cases have occurred. We propose a hypothesis test in which the basic idea is to reject $\mathrm{H}_{0}$ if the number of days that have elapsed since the occurrence of the $n^{\text {th }}$ case, called $T$, is sufficiently large, say greater than $t$. We choose $t$ to control the type 1 error probability based on the $n^{\text {th }}$ spacing. The probability that the $n^{\text {th }}$ spacing is greater than $t$ is

$$
\mathrm{P}\left(s_{n}>t\right)=\exp (-\lambda(N-n) t)
$$

We can use equation (1) to perform a significance test at level $\alpha$. We choose $t$ such that (2) is equal to $\alpha$ at the particular null hypothesis when $N=n+1$. Then, it follows from (2) that we reject $\mathrm{H}_{0}$ when the elapsed time since the occurrence of the last case, $T$, is such that

$$
T \geq-\mu \ln (\alpha)
$$

where $\mu=\lambda^{-1}$. At other values of $N$ under the null hypothesis, that is if $N>n+1$, we can be assured that (2) will be less than $\alpha$, and that is because the right side of (2) decreases as $N$ increases. Thus, as is generally done with one-sided composite null hypotheses we focus on the boundary point between the null and alternative hypotheses, which in this case is $N=n+1$ (Cox 
and Hinkley, 1974). In general for any $N>n$, it follows from using (1) and (2) that the probability of rejecting $\mathrm{H}_{0}$ is

$$
P\left(s_{n} \geq-\mu \ell \mathrm{n}(\alpha)\right)=\mathrm{e}^{\mu(N-n) \frac{\ell \mathrm{n} \alpha}{\mu}}=\alpha^{(N-n)}
$$

In practice, since we do not know the value of $\mu$, we propose inserting an estimate $\hat{\mu}$ for $\mu$ in (3). For example, under the exponential model from a sample of size $N$, the spacings are independent, and the maximum likelihood estimator based on the first $n$ - 1 spacings can be shown to be:

$$
\hat{\mu}=\frac{\sum_{j=1}^{n-1} s_{j}(N-j)}{n-1}
$$

and $\hat{\mu}=\hat{\lambda}^{-1}$. This estimator involves the unknown $N$. In practice, we propose replacing $N$ by the particular value under $\mathrm{H}_{0}$ when $N=n+1$. When $n+1$ is substituted for $N$ in (5), we call the resulting estimator $\hat{\mu}_{0}$. The proposed hypothesis test procedure is to reject $\mathrm{H}_{0}$ if the duration of time that has elapsed since the last case, $T$, is sufficiently large, specifically, we reject $\mathrm{H}_{0}$ if

$$
T \geq-\hat{\mu}_{0} \ell \mathrm{n}(\alpha)
$$

However, the probability of a type 1 error-based on (6) will now be greater than $\alpha$ because $\mu$ is being replaced by an estimate. Let the probability of a type 1 error when $\mu$ is replaced by an estimator be $\tilde{\alpha}=P\left(S_{n}>-\hat{\mu}_{0} \ell\right.$ ln $\left.\alpha\right)$. We now show how to estimate $\tilde{\alpha}$ under $\mathrm{H}_{0}$ as follows. The approach is to condition on a fixed value for $\hat{\mu}_{0}$ and, second, to integrate over its sampling distribution $g\left(\hat{\mu}_{0}\right)$. That conditional probability is $P\left(S_{n}>-\hat{\mu}_{0} \ell n \alpha \mid \hat{\mu}_{0}\right)=\exp \left(\frac{\hat{\mu}_{0}(N-n) \ell n \alpha}{\mu}\right)=\alpha^{(N-n) \hat{\mu}_{0} / \mu}$. Thus 


$$
\tilde{\alpha}=\int \alpha^{(N-n) \hat{\mu}_{0} / \mu} g\left(\hat{\mu}_{0}\right) d \hat{\mu}_{0}
$$

We approximate the first factor in the integrand of (7) by a second order Taylor series expansion about $\hat{\mu}_{0}=\mu$ and obtain,

$$
\alpha^{(N-n) \hat{\mu}_{0} / \mu} \approx \alpha^{N-n}+\frac{\alpha^{N-n}(N-n) \ell n \alpha\left(\hat{\mu}_{0}-\mu\right)}{\mu}+\frac{\alpha^{N-n}}{2}\left(\frac{(N-n) \ell n \alpha}{\mu}\right)^{2}\left(\hat{\mu}_{0}-\mu\right)^{2} .
$$

Now, consider the particular null hypothesis when $N=n+1$. Substituting the above expression into (7), using a large sample approximation that $\hat{\mu}_{0}$ is nearly unbiased estimator of $\mu$ when $N=n+1$ and that $\operatorname{Var}\left(\hat{\mu}_{0}\right) \approx \mu^{2} /(n-1)$, we obtain

$$
\tilde{\alpha} \approx \alpha^{N-n}\left[1+\frac{(N-n)^{2}(\ell n \alpha)^{2}}{2(n-1)}\right]
$$

It follows that $\tilde{\alpha}$ is always greater than $\alpha$. It can also be shown that (8) is a good approximation for other values of $N$ under $\mathrm{H}_{0}$, that is when $N>n+1$. Furthermore, the right hand side of (8) decreases as $N$ increases. Thus, the right hand side of equation (8) with $n+1$ substituted for $N$ would be conservative in that it overestimates the probability of a type 1 error for all values of $N$ under $\mathrm{H}_{0}$.

To see how to use equation (8) in practice, suppose we want the probability of a type 1 error to be no greater than .05 for all values of $N$ under $\mathrm{H}_{0}$ and we have observed $n$ cases. Then we can set equation (8) equal to .05 with $N=n+1$ and solve for $\alpha=\alpha_{n, 05}$ (where we have added subscripts to emphasize that the solution for $\alpha$ depends on $n$ ):

$$
.05=\alpha_{n, 05}\left[1+\frac{\left(\ln \alpha_{n, 05}\right)^{2}}{2(n-1)}\right]
$$


Figure 1 shows the values $\alpha_{n, 05}$ to be substituted for $\alpha$ in (3) to insure that the type 1 error probability is no greater than .05 . For example, if $n=20$ cases have occurred and we wish the type 1 error probability to be .05 , then $\alpha_{n, 05}=.039$, and thus we should set $\alpha=.039$ in equation

(3). Figure 1 also shows values for $\alpha_{n, 10}$.

\subsection{Comments on the Hypothesis Test}

Let's consider first the special situation when $\mu$ is known. In that situation, equation 3 implies that we should reject the null hypothesis at level $\alpha$ if the spacing between cases becomes greater than $-\mu \ln \alpha$. Thus, we reject $\mathrm{H}_{0}$ at the .05 level if the elapsed time since the last case is at least a factor of $-\ln (.05) \approx 3$ times greater than the mean incubation period. For example, if the mean incubation period is 10 days, then at level .05 we would reject the null hypothesis if the spacing between cases became greater than $t=30$ days. If $\mu$ is unknown, then the specific value of $t$ depends on $n$. For example for $n=20$ and 40, we would reject $\mathrm{H}_{0}$ at the .05 level if the elapsed time since the last case is at least a factor of 3.1 and 3.2 times greater than the estimated mean incubation period, respectively (these factors are $-\ln \left(\alpha_{n, 05}\right)$ where $\alpha_{n, .05}$ is calculated from equation 9 and displayed in Figure 1).

The calculations in the previous paragraph suggest that the elapsed time necessary to declare the end of an epidemic is surprisingly long. However, the outbreak can be declared “almost" over with considerably shorter periods of time. To make this notion concrete, consider the hypothesis that there will be no more then $r$ additional cases. The null and alternative hypotheses are then:

$$
\begin{aligned}
& \mathrm{H}_{0}: N>n+r \\
& \mathrm{H}_{1}: N \leq n+r
\end{aligned}
$$


The approach is to reject $\mathrm{H}_{0}$ if the elapsed time since the last case, $T$, is sufficiently large, say $c$ times greater than $\mu$. Then for an $\alpha$-level test, $c$ is chosen such that $\mathrm{P}\left(\mathrm{S}_{n}>c \mu\right)=\alpha$. It follows from equation 4, that $\mathrm{P}\left(\mathrm{S}_{n}>c \mu\right)=\exp (-c(N-n))$ which is set equal to $\alpha$ with $N=n+r+1$ and we solve for c. We find we reject $H_{0}$ if

$$
T \geq-\frac{\mu \ln \alpha}{r+1}
$$

Thus, if we wanted to test the hypothesis that there will be no more than $r=1,2$ or 3 additional cases, then we would reject $\mathrm{H}_{0}$ at the .05 level if the elapsed time since the last case was respectively at least a factor of $1.5,1.0$ and 0.75 greater than $\mu$. If $\mu$ is unknown and is estimated from the data, similar findings also apply. Specifically, we can solve for $c$ by setting equation 8 equal to the desired level of significance, say for example .05, and we solve for $\alpha_{\mathrm{n}, 05}$ with $N=n+r+1$. Then, $c=-\ln \alpha_{n, 05}$. For example, if $n=20$ and we wanted to test the hypothesis that there will be no more than $r=1,2$ or 3 additional cases, we calculate that we reject $\mathrm{H}_{0}$ at the .05 level if the elapsed time since the last case was respectively at least a factor of $1.62,1.08$ and 0.81 times greater than the estimated mean incubation period.

\section{Outbreak-wise Error Rates}

As an outbreak unfolds in real time, public health officials may wish to repeatedly test whether the outbreak has ended after successive cases. The overall outbreak-wise error rate should be larger than that of a single hypothesis test at a single point in time because of the multiple hypothesis testing. We investigated the impact of multiple hypotheses testing on the overall outbreak-wise type 1 error rate.

Suppose a hypothesis test is performed on each spacing beginning with the $k^{\text {th }}$ spacing with type 1 error of no greater than $\alpha$ on each individual test. Then, the overall probability of prematurely declaring the outbreak has ended is 


$$
\rho=1-\prod_{n=k}^{N-1}\left(1-\tilde{\alpha}{ }_{n}\right)
$$

where $\tilde{\alpha}_{n}$ is the probability of a type 1 error from a hypothesis test based on the $n^{\text {th }}$ spacing (we have added a subscript $n$ to emphasize that $\tilde{\alpha}_{n}$ depends on the number of observed cases.) First, consider the special case, when $\mu$ is known. From equation (4) we have $\tilde{\alpha}_{n}=\alpha^{(N-n)}$. Inserting this expression for $\tilde{\alpha}_{n}$ into the expression for $\rho$, we find that the outbreak-wise error rate is $\rho \leq \alpha+\alpha^{2}$ for all $N$ and all values of $k$. This is a surprising result because it says that the additional penalty for multiple testing with respect to the overall type 1 error is quite small because $\alpha^{2}$ is negligible compared to $\alpha$. For example, if each individual hypothesis test is performed at level $\alpha=.05$, then the outbreak-wise error rate is .0525 regardless of the size of the outbreak. The intuition for this result is that even though more tests are performed with larger $N$, the actual type 1 error probability on the $n^{\text {th }}$ test $\left(\tilde{\alpha}_{n}\right)$ decreases as $N$ increases with $n$ fixed. The source of most of the type 1 errors occurs at the hypothesis test for the last spacing; tests on earlier spacings contribute relatively few additional type 1 errors.

We then investigated the outbreak-wise error rate when $\mu$ is unknown and is estimated from the data on spacings. For example, inserting $\alpha_{n, 05}$ for $\alpha$ in equation (6) guarantees that the type 1 error probability is no greater that .05 on the test of the $n^{\text {th }}$ spacing for all values of $N$ under the null hypothesis (that is, $N>n$ ). In order to compute the type 1 error probability from the test on the $n^{\text {th }}$ spacing, we insert $\alpha_{n, 05}$ for $\alpha$ in equation 8 , and obtain

$$
\tilde{\alpha}_{n}=\left(\alpha_{n, 05}\right)^{N-n}\left[1+\frac{(N-n)^{2}\left(\ln \alpha_{n, 05}\right)^{2}}{2(n-1)}\right]
$$

Table 1 shows the outbreak-wise error rates $\rho$ that were calculated using the above expression for $\tilde{\alpha}_{n}$ when tests are performed on all spacings beginning with the $k=5^{\text {th }}$ spacing. For example, 
suppose $N=30$ and each individual test is performed using $\alpha_{n, 05}$ to insure that the type 1 error probability is not greater than .05 on each individual test; then, as shown in Table 1 the outbreak-wise type 1 error rate is .053 . Thus, multiple hypotheses testing throughout the course of an outbreak have a very minor effect on inflating the overall outbreak-wise type 1 error rates even in the case when $\mu$ is estimated.

[Table 1 here]

\section{Simulation Study}

A simulation study was performed to evaluate the performance of the hypothesis testing procedures described in section 2. The methods in section (2) are based on an underlying exponential distribution with a guarantee time. Furthermore, equation (9) is based on a large sample approximation. The simulation study allows us to evaluate the robustness of the methods to violations of the exponential assumption and their performance in small or moderate sample sizes. Outbreaks of size $N=15,20,40$ and 60 were generated using various incubation period distributions. We performed 1000 simulated outbreaks for each set of conditions. The cases of disease were ordered by their dates of occurrences, the mean incubation time was estimated and hypothesis tests were performed. We calculated the empirical error rate of falsely declaring the epidemic is over based on the $(N-1)^{\text {st }}$ spacing using equation (6) with $\alpha=\alpha_{n}, .05$. We also calculated the outbreak-wise error rates when testing begins at the $5^{\text {th }}$ spacing and continues to the last spacing.

The underlying incubation period distributions that we used in the simulation study included the exponential model and the lognormal model. The lognormal model for the incubation period of infectious diseases has been widely used. Sartwell (1950) for example, applied the lognormal model to 18 data sets representing 13 different infectious diseases including measles and 
salmonelliosis. Sartwell defined the dispersion parameter in the lognormal model as $d=\exp (\sigma)$ where $\sigma$ is the standard deviation of the logarithm of the incubation periods. The interpretation of the dispersion parameter is that the probability is .69 that the incubation period will fall between the median incubation period divided by $d$ and the median multiplied by $d$. Sartwell found that although the median incubation periods were very different across a wide variety of diseases the dispersion parameters were similar ranging between 1.1 and 2.1. Motivated by this work, we used a low dispersion factor of $1.1(\sigma=.1)$, moderate dispersion of $1.6(\sigma=.6)$, and a high dispersion of $2.2(\sigma=0.8)$.

The simulation results are shown in Table 2. The empirical error rates for the $(\mathrm{N}-1)^{\mathrm{st}}$ spacing should be approximately .05 . The outbreak wise error rates should be just slightly larger than .05 as previously shown in Table 1 . We found that when the underlying model is exponential, the empirical error rates are close to the nominal levels for outbreaks as small as $\mathrm{N}=20$ and regardless of the value of $\mu$. When the underlying model is lognormal, the empirical error rates depend critically on the value of the dispersion parameter $d$. Generally, the empirical error rates are smaller than predicted for small and moderate values of the dispersion parameter. However, in the case of high dispersion, we found that the empirical error rates are greater than expected and the methods are anticonservative.

[Table 2 here]

\section{Discussion}

The objective of this paper was to develop a hypothesis test for the end of a common source outbreak if neither the date of exposure to the pathogen nor the specific incubation period distribution of disease is known. The proposed test is based on the spacings between cases which help circumvent the difficulty of not knowing the date of exposure. The method provides 
a theoretical framework for understanding ad-hoc methods used by public health practitioners based on the elapsed time since the occurrence of the latest case.

One surprising implication of our methodology is that the elapsed time since the latest case must be quite long before one can confidently declare that an outbreak has ended. As discussed in section 2.3, the elapsed time since the latest case must be at least a factor of 3 times greater than the mean incubation period to declare the outbreak over at the .05 level of significance. In the 2001 U.S. anthrax outbreak some public health officials had suggested that if 7 days had elapsed with no new cases, then that was good evidence that the outbreak has ended. However, the mean incubation period of anthrax has been estimated to be 14 days, and thus a 7 day period in which no new cases have been reported is too short to confidently declare the end of an outbreak. Our analysis serves as a cautionary note about prematurely declaring an outbreak over based on spacings of moderate length. However, as discussed in section.2.3, our analysis also shows that periods of moderate duration in which no new cases have occurred (e.g. about equal to the mean incubation period) can signal the outbreak is "almost" over, that is, there are only a few remaining cases still to occur. Our analysis also shows that the penalty on the type 1 error probability for multiple testing during the course of an outbreak is very minor.

The methods we derived in section 2 assumed a two parameter exponential model with guarantee time for the incubation period distribution. The performance of the methods with respect to type 1 error rates was good when the underlying incubation distribution was exponential. We evaluated the robustness of the methods to the lognormal model for the incubation period distribution. We found that the performance depends critically on the dispersion parameter of the lognormal model. Generally, the empirical type 1 error rates were equal or smaller than the nominal levels for the range of dispersion parameters typically found in 
infectious diseases $(d<2.1)$ (Sartwell, 1950). However, if the dispersion parameter was greater, the methods will no longer be conservative, and one could certainly not rule out the possibility that new and emerging pathogens might exhibit dispersion greater than seen in other infectious diseases. One area of future work is semi-parametric methods that could take advantage of the fact that spacings are asymptotically exponentially distributed regardless of the parametric model (Cox and Hinkley, 1974); however estimation of the mean of that exponential distribution would require density estimation, and some critical assumptions would still need to be made about the tail of the incubation distribution.

Considerable caution is required if the incubation period distribution deviates substantially from either the exponential or lognormal models, and in particular if the incubation period distribution is not unimodal. For example, suppose the incubation period distribution was bimodal arising from a mixture of 2 lognormal distributions corresponding to one subpopulation with a small median incubation period and one subpopulation with a large median incubation period. Then, a long period of time in which no new cases occur could mistakenly signal the end of the outbreak when instead it signals the end of cases from the first subpopulation, with no indication that a second wave of cases is about to occur from the second subpopulation. A multi-modal distribution could also arise if there was not a single calendar date of exposure. For example, outbreaks such as those associated with contaminated food may have multiple dates of exposure if the food had been distributed to multiple locations. Such phenomena could create multiple waves of an outbreak.

The methods may provide useful guidance to public health practitioners facing the challenge of making forecasts about the course of a common source outbreak from dates of occurrence of cases with no additional epidemiological information. However, as additional epidemiological 
information becomes available during the course of an outbreak, such as the dates of exposure or the incubation period distribution, such information could and should be incorporated into the methods to provide more reliable forecasts. 


\section{ACKNOWLEDGEMENTS}

This work was partially supported by a grant from the National Institute of Allergy and Infectious Diseases 
Figure 1. Values of $\alpha_{n, 05}$ and $\alpha_{n, 10}$, versus $n$. Rejecting $\mathrm{H}_{0}$ when $T>-\hat{\mu}_{0} \ell n \alpha_{n, \alpha}$ insures that the type 1 error probability will be no greater than $\alpha$. 


\section{Table 1}

Outbreak-wise error rates versus $N$ and type 1 error rate $(\tilde{\alpha})$ on each individual test. Outbreakwise probabilities of type 1 error are based on testing each spacing in an outbreak beginning with the $5^{\text {th }}$ spacing.

\begin{tabular}{cccc}
\hline \hline $\mathbf{N}$ & & $\tilde{\alpha}$ & \\
\hline & .01 & .05 & $\mathbf{1 0}$ \\
10 & .0101 & .0530 & .1134 \\
20 & .0101 & .0533 & .1127 \\
40 & .0101 & .0530 & .1115 \\
100 & .0101 & .0527 & .1106 \\
\hline
\end{tabular}


Table 2

Simulation results of type 1 error rates for hypothesis test of the end of a common-source outbreak (each based on 1000 simulations; each hypothesis test performed with $\alpha$ in equation (6) set to $\alpha_{n, 05}$ ). Outbreak-wise empirical error rate based on testing each spacing beginning with the $5^{\text {th }}$ spacing and ending with $(N-1)^{\text {st }}$ spacing.

\begin{tabular}{|c|c|c|c|}
\hline \multirow[b]{2}{*}{ Distribution } & \multirow[b]{2}{*}{$\mathrm{N}$} & \multicolumn{2}{|c|}{ Empirical error rates } \\
\hline & & $(\mathrm{N}-1)^{\mathrm{st}}$ & Outbreak-wise \\
\hline Exponential $(\mu=5)$ & 15 & .061 & .086 \\
\hline Exponential $(\mu=10)$ & 15 & .039 & .067 \\
\hline Exponential $(\mu=5)$ & 20 & .055 & .077 \\
\hline Exponential $(\mu=10)$ & 20 & .056 & .075 \\
\hline Exponential $(\mu=5)$ & 40 & .058 & .065 \\
\hline Exponential $(\mu=10)$ & 40 & .043 & .051 \\
\hline Exponential $(\mu=5)$ & 60 & .047 & .052 \\
\hline Exponential $(\mu=10)$ & 60 & .053 & .059 \\
\hline Lognormal $(d=1.1)$ & 15 & .000 & .001 \\
\hline $\operatorname{Lognormal}(d=1.8)$ & 15 & .036 & .043 \\
\hline $\operatorname{Lognormal}(d=2.2)$ & 15 & .107 & .133 \\
\hline $\operatorname{Lognormal}^{1}(d=1.1)$ & 20 & .000 & .000 \\
\hline Lognormal $(d=1.8)$ & 20 & .044 & .049 \\
\hline Lognormal $(d=2.2)$ & 20 & .105 & .122 \\
\hline Lognormal $(d=1.1)$ & 40 & .000 & .001 \\
\hline $\operatorname{Lognormal}(\mathrm{d}=1.8)$ & 40 & .047 & .051 \\
\hline Lognormal $(\mathrm{d}=2.2)$ & 40 & .127 & .146 \\
\hline Lognormal $(d=1.1)$ & 60 & .000 & .000 \\
\hline $\operatorname{Lognormal}(d=1.8)$ & 60 & .059 & .064 \\
\hline Lognormal $(d=2.2)$ & 60 & .151 & .182 \\
\hline
\end{tabular}

${ }^{1}$ Median incubation time for lognormal models was $\exp (2.5)=12.2$ 


\section{REFERENCES}

Brookmeyer, R. and Blades, N. (2002). Prevention of inhalational anthrax in the U.S. outbreak. Science 295, 1861.

Brookmeyer, R., Blades, N., Hugh-Jones, M. et al. (2001). The statistical analysis of truncated data: application to the Sverdlovsk anthrax outbreak. Biostatistics 2, 233-247.

Brookmeyer, R. and Gail, M. H. (1994). AIDS Epidemiology: A Quantitative Approach. London: Oxford University Press.

Brookmeyer, R., Johnson, E. and Barry, S. (2004). Modeling the incubation period of anthrax. Statistics in Medicine (in press).

Brookmeyer, R., Johnson, E. and Bollinger, R. (2003). Modeling the optimum duration of antibiotic prophylaxis in an anthrax outbreak. Proceedings of the National Academy of Sciences 100(17), 10129-10132.

Cox, D. R. and Hinkley, D.V. (1974). Theoretical Statistics. London: Chapman and Hall.

David, H.A. (1970). Order Statistics. New York: John Wiley and Sons.

Giesbrecht, F. and Kempthorne, O. (1976). Maximum likelihood estimation in the threeparameter lognormal distribution. Journal of the Royal Statistical Society, Series B 38, 257-264.

Hill, B. M. (1963). The three-parameter lognormal distribution and Bayesian analysis of a point source epidemic. Journal of the American Statistical Association 58, 72-84.

Pike, R. (1965). Spacings (with discussion). Journal of the Royal Statistical Society, Series B 27, 395-449.

Sartwell, P. E. (1950). The distribution of incubation periods of infectious diseases. American Journal of Hygiene 51, 310-318. 
Walden, J. and Kaplan, E. (2004). Estimating time and size of bioterror attack. Emerging Infectious Diseases 10(7). 


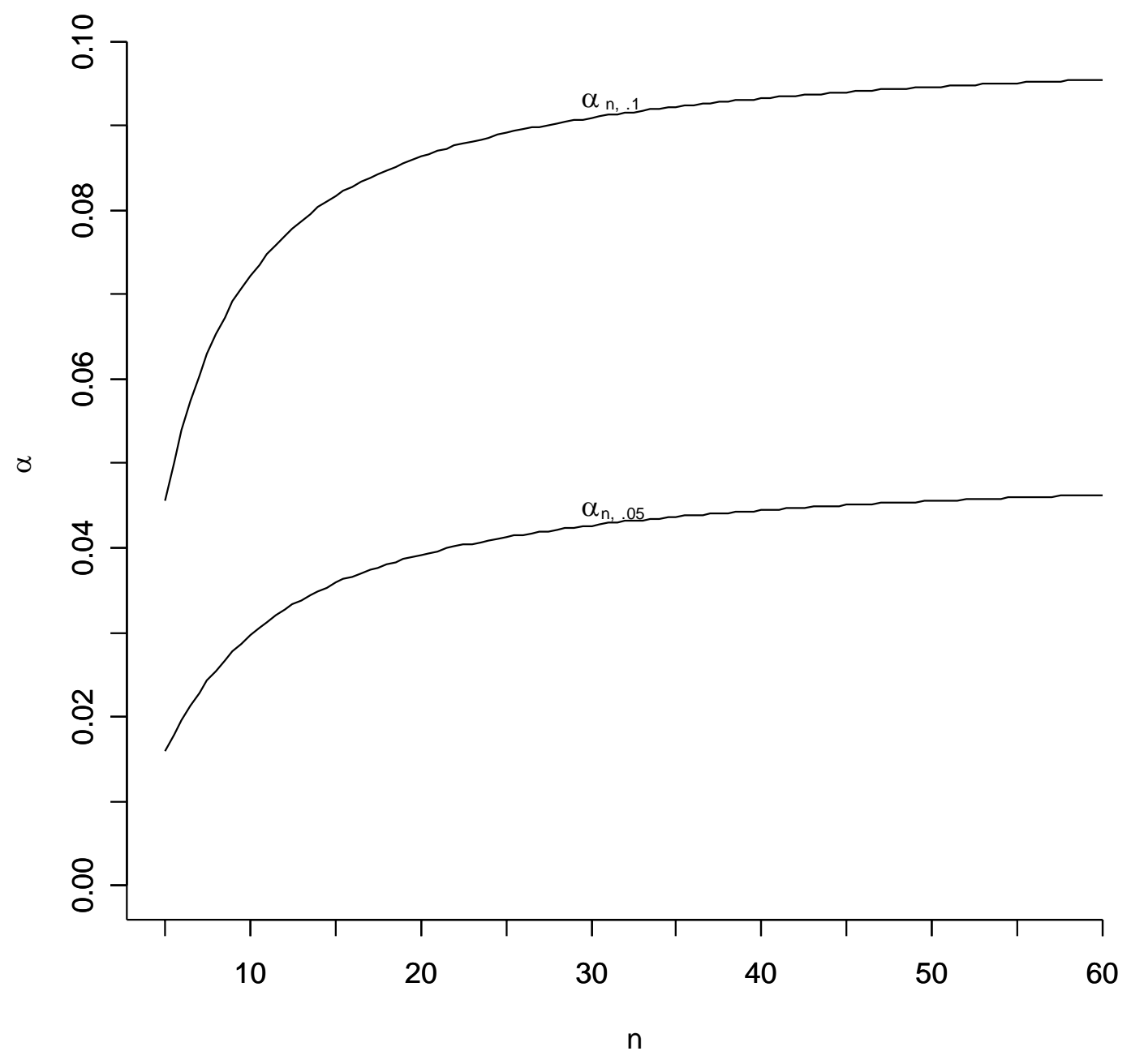

